\title{
Monte Carlo evaluation of the equilibrium isotope effects using the Takahashi-Imada factorization of the Feynman path integral
}

\author{
Marcin Buchowiecki \\ Institute of Physics, University of Szczecin, Wielkopolska 15 \\ Szczecin, 70-451, Poland \\ mabuch@wmf.univ.szczecin.pl \\ Jiří Vaníček \\ Laboratory of Theoretical Physical Chemistry, Institut des Sciences et Ingénierie \\ Chimiques, École Polytechnique Fédérale de Lausanne (EPFL), CH-1015 Lausanne, \\ Switzerland \\ jiri.vanicek@epfl.ch
}

\begin{abstract}
The Feynman path integral approach for computing equilibrium isotope effects and isotope fractionation corrects the approximations made in standard methods, although at significantly increased computational cost. We describe an accelerated path integral approach based on three ingredients: the fourthorder Takahashi-Imada factorization of the path integral, thermodynamic integration with respect to mass, and centroid virial estimators for relevant free energy derivatives. While the first ingredient speeds up convergence to the quantum limit, the second and third improve statistical convergence. The combined method is applied to compute the equilibrium constants for isotope exchange reactions $\mathrm{H}_{2}+\mathrm{D} \rightleftharpoons \mathrm{H}+\mathrm{HD}$ and $\mathrm{H}_{2}+\mathrm{D}_{2} \rightleftharpoons 2 \mathrm{HD}$.
\end{abstract}

Keywords: path integral Monte Carlo, Takahashi-Imada factorization, equilibrium isotope effect

\section{Introduction}

The equilibrium isotope effect (EIE), defined as the effect of isotopic substitution on chemical equilibrium, is amongst the most important tools 
for studying reaction mechanisms and equilibria [1]. In certain situations, e.g., in composite mechanisms with a pre-equilibrium, the EIE can be the main determinant of the kinetic isotope effect. Although EIE research has a long history, until recently most theoretical calculations were based on the assumption of separability of rotations and vibrations, on the rigid rotor approximation for rotations, and on the harmonic treatment of vibrations [1]. More rigorous EIE treatments avoiding these three approximations are recent [2, 3, 4, 5, 6, 7, 8] and based on the Feynman path integral [9, 10, 11] representation of the partition function.

One of these rigorous approaches [3], the extension of which is the main goal of this article, evaluates the required ratio of partition functions via thermodynamic integration with respect to the isotope mass (used originally [12, 13, 14] in the context of quantum instanton [15] calculation of kinetic isotope effects) and via Monte Carlo sampling of the discretized path integral. The method of Ref. [3] was successfully used with ab initio potentials for calculating the EIE in $[1,5]$ sigmatropic hydrogen shift reactions [3, 4]. The computational cost was drastically lowered by combining the harmonic estimate of the EIE based on accurate ab initio electronic structure calculations together with path integral Monte Carlo simulations based on force fields or semiempirical potentials for calculating the anharmonicity and other quantum corrections. In both Refs. [3, 4], only the standard primitive approximation for the path integral was used.

Lynch, Mielke, and Truhlar [2] evaluated accurate equilibrium isotope effects on $\mathrm{H}_{2} \mathrm{O}_{2}$ as ratios of partition functions computed with the enhanced same-path extrapolation variant [16] of the trapezoidal Trotter Fourier path integral Monte Carlo method [17]. Another method, proposed by Major and coworkers [5] combines the quantized classical path approach of Hwang and Warshel [18] with a perturbation expression using the staging algorithm. After comparing implementations with the primitive, Takahashi-Imada [19], and Chin [20] factorizations of the discretized path integral, the authors applied their method to compute the EIE on the keto-enol tautomerism in alanine racemase [5]. Perez and von Lilienfeld generalized the calculation of EIEs using "alchemical" transformations in which the isotope masses are changed simultaneously with the interaction potential [6]. Ceriotti and Manolopoulos sped up EIE calculations by combining path integral molecular dynamics with a generalized Langevin equation [7]. Very recently, Ceriotti and Markland accelerated the thermodynamic integration with respect to mass by "flattening" the integral with a nonlinear change of variables [8]. 
In general, methods based on path integrals are accurate, but also very computationally expensive when compared with the less rigorous approaches. Consequently, any extension that significantly speeds up the convergence of path integral simulations without compromising accuracy is highly desirable. The aim of this paper is to accelerate the convergence of the Ref. [3] method to the quantum limit through combination with the fourth-order Takahashi-Imada factorization [19] of the path integral. The smaller error in Takahashi-Imada factorization allows discretization of the path integral with fewer imaginary time slices than required by the primitive approximation used in Ref. [3]. In addition to lowering the systematic error by the Takahashi-Imada factorization, we also decrease the statistical error by implementing the centroid virial estimator for the free energy derivative with respect to mass and specific for the Takahashi-Imada factorization. The improved methodology is tested on two isotope exchange reactions on the BKMP2 potential energy surface [21]. In contrast to Ref. [3], where the discretized path integral was sampled with the path integral molecular dynamics, here the sampling is performed with the path integral Monte Carlo method.

The remainder of the article is organized as follows: Section 2 explains how to evaluate EIEs via the thermodynamic integration, describes the implementation of this method using the Takahashi-Imada factorization of the path integral, and presents the derivation of relevant thermodynamic and centroid virial estimators. The methodology described in Sec. 2 is applied to two different chemical reactions in Sec. 3, while Sec. 4 concludes the paper.

\section{Theory}

\subsection{Thermodynamic integration with respect to mass}

The EIE is defined as the ratio EIE $=K_{l} / K_{h}$, where $K_{l}$ and $K_{h}$ are the equilibrium constants of two chemical reactions that involve lighter $(l)$ and heavier $(h)$ isotopologs, but are otherwise identical. In terms of the molecular partition functions for the products $(P)$ and reactants $(R)$, the EIE is expressed as [22]

$$
\mathrm{EIE}=\frac{Q_{l}^{P} / Q_{l}^{R}}{Q_{h}^{P} / Q_{h}^{R}} .
$$

A more convenient form of the last expression, namely,

$$
\mathrm{EIE}=\frac{Q_{h}^{R} / Q_{l}^{R}}{Q_{h}^{P} / Q_{l}^{P}},
$$


suggests that an evaluation via the thermodynamic integration [23] with respect to the isotopic mass is possible [3, 13]. In this method, the ratios of partition functions in Eq. (2) are computed from the reduced free energy difference $\Delta F$ as

$$
\frac{Q_{h}}{Q_{l}}=\frac{s_{l}}{s_{h}} \exp (-\beta \Delta F),
$$

where $s_{l}$ and $s_{h}$ denote the symmetry numbers of the two isotopologs and $\beta=1 /\left(k_{B} T\right)$ is the inverse temperature. By factoring out the symmetry numbers, we take into account the exchange symmetry of the indistinguishable hydrogen nuclei approximately and $\Delta F$ can be computed as for distinguishable particles. The symmetry numbers reflect the number of indistinguishable orientations of the molecule in which hydrogen atoms are exchanged by rotation [1, 22, 24]. The value of EIE stemming from the symmetry numbers alone is referred to as a purely statistical isotope effect, which is equal to the high temperature approximation described below. The treatment of indistinguishability by symmetry factors is valid even at temperatures much lower than those considered here [9, 11]. (To go beyond this approximation is possible, but requires Feynman paths connecting different particles [9, 11]; the contributions of such paths to the Feynman path integral are strongly suppressed except at temperatures close to absolute zero.)

The reduced free energy difference $\Delta F$ is evaluated as the integral

$$
\Delta F=\int_{0}^{1} d \lambda \frac{d F(\lambda)}{d \lambda}
$$

of the derivative of $F$ with respect to a parameter $\lambda$ interpolating between the masses of isotopes in the two isotopologs. For convenience, we choose this interpolation to be linear:

$$
m_{i}(\lambda)=(1-\lambda) m_{l, i}+\lambda m_{h, i}
$$

Finally, note that for an isotopomerization reaction (a reaction in which $R$ and $P$ are isotopomers, i.e., involve the same numbers of all isotopes), the EIE can be simply defined as the equilibrium constant: EIE $=K=Q^{P} / Q^{R}$.

\subsection{Monte Carlo evaluation of the free energy derivatives via the Takahashi-} Imada factorization of the path integral

The path integral formalism [9, 10, 11] allows for a rigorous representation of the quantum partition function $Q(\beta)=\operatorname{Tr}[\exp (-\beta \hat{H})]$ without knowledge 
of the eigenstates of the Hamiltonian operator. Using the Lie-Trotter theorem [25], the trace on the right side of this expression can be factorized as

$$
\operatorname{Tr}\left(e^{-\beta \hat{H}}\right)=\operatorname{Tr}\left\{\left[e^{-(\beta / P) \hat{T}} e^{-(\beta / P) \hat{V}}\right]^{P}\right\}+\mathcal{O}\left(\beta^{3} P^{-2}\right),
$$

where $\hat{T}$ and $\hat{V}$ are the kinetic and potential energy operators, and $P$ is the Trotter number (or number of imaginary time slices or "beads"). Setting $P=1$ in Eq. (51) yields the classical partition function, whereas the limit $P \rightarrow \infty$ gives the exact quantum partition function. Factorization (5) is usually called the primitive approximation (PA). Due to its simplicity, it is the most widely used path-integral factorization and was used for the calculation of the EIEs in Ref. [3].

Our main goal is to demonstrate that the convergence of the EIE calculation with respect to $P$ is significantly accelerated by employing the Takahashi-Imada (TI) factorization [19],

$$
\operatorname{Tr}\left(e^{-\beta \hat{H}}\right)=\operatorname{Tr}\left\{\left[e^{-(\beta / P) \hat{T}} e^{-(\beta / P) \hat{V}_{\text {eff }}}\right]^{P}\right\}+\mathcal{O}\left(\beta^{5} P^{-4}\right)
$$

where $\hat{V}_{\text {eff }}$ is an effective potential energy operator obtained by augmenting the true potential energy $\hat{V}$ with a TI term $\hat{V}_{\mathrm{TI}}$,

$$
\begin{aligned}
\hat{V}_{\text {eff }} & =\hat{V}+\hat{V}_{\text {TI }}, \\
\hat{V}_{\text {TI }} & =\frac{1}{24}\left(\frac{\beta}{P}\right)^{2}[\hat{V},[\hat{T}, \hat{V}]] .
\end{aligned}
$$

In the coordinate representation,

$$
V_{\mathrm{TI}}(\mathbf{r})=\frac{1}{24} \hbar^{2}\left(\frac{\beta}{P}\right)^{2} \sum_{i=1}^{N} \frac{1}{m_{i}}\left(\frac{\partial V}{\partial \mathbf{r}_{i}}\right)^{2} .
$$

where $r=\left(r_{1}, \ldots, r_{N}\right)$ is a collective notation for the coordinates of all $N$ atoms. Having accuracy of higher order in $P$, the TI factorization is more accurate than the PA for a given (sufficiently large) $P$. As a consequence, the TI factorization achieves a desired accuracy with a smaller Trotter number $P$ than the PA. This is an advantage, particularly at low temperatures, where the Trotter number required for simulation convergence with the PA can be very large [5, 19, 26, 27, 29, 30]. 
Both PA (5) and TI factorization (6) are expressed exactly in the coordinate representation. In the TI scheme,

$$
Q_{\mathrm{TI}, P}(N, V, T)=C \int d\left\{\mathbf{r}^{(s)}\right\} \exp \left[-\beta \Phi_{\mathrm{eff}}\left(\left\{\mathbf{r}^{(s)}\right\}\right)\right]
$$

where $\left\{\mathbf{r}^{(s)}\right\}$ stands for $\left(\mathbf{r}^{(1)}, \ldots, \mathbf{r}^{(P)}\right)$; the prefactor $C$ and effective "polymer chain" potential $\Phi_{\text {eff }}$ are

$$
\begin{aligned}
C & =\left(\frac{P}{2 \pi \hbar^{2} \beta}\right)^{3 N P / 2} \prod_{i=1}^{N} m_{i}^{3 P / 2} \text { and } \\
\Phi_{\mathrm{eff}}\left(\left\{\mathbf{r}^{(s)}\right\}\right) & =\frac{P}{2 \hbar^{2} \beta^{2}} \sum_{i=1}^{N} m_{i} \sum_{s=1}^{P}\left(\mathbf{r}_{i}^{(s)}-\mathbf{r}_{i}^{(s+1)}\right)^{2} \\
& +\frac{1}{P} \sum_{s=1}^{P} V_{\mathrm{eff}}\left(\mathbf{r}^{(s)}\right) .
\end{aligned}
$$

The discretized path integral (9) is evaluated with either the path integral Monte Carlo (PIMC) or path integral molecular dynamics, with both approaches having advantages and disadvantages. While the path integral molecular dynamics appears to be the method of choice for ab initio path integral calculations [29], its PA implementation requires force and energy evaluations, whereas the PIMC needs only energies. The TI factorization implemented in a PIMC code requires only energies and forces, whereas for path integral molecular dynamics energies, forces, and second derivatives of the energies must be calculated. The second derivative requirement was circumvented by Jang and coworkers [26], Yamamoto [28], and Perez and Tuckerman [29] with an elegant trick employing the PA as a reference potential; however, this assumes that the TI correction (8) is small. Ceriotti and coworkers [31] showed recently that this re-weighting procedure fails in systems with many degrees of freedom due to the growth of the statistical error of the re-weighted average.

Therefore we focus on the PIMC implementation of the TI factorization: In addition to not requiring second derivatives of the potential energy, its implementation into existing PIMC codes is straightforward, the main change being that both the random walk and the estimators use $\Phi_{\text {eff }}$ instead of the $\Phi$ originally employed in the PA. Below we list only the new, TI expressions; the 
corresponding PA expressions can be obtained by setting $V_{\mathrm{TI}}=0$. Moreover, they have already been derived in Ref. [14].

PIMC simulations [11] are based on the sampling of the full $3 N P$-dimensional configuration space of the discretized path-integral representation (9) of the partition function. The polymer-chain potential energy $\Phi_{\text {eff }}$ from Eq. (10) determines the sampling weight $W=\exp \left(-\beta \Phi_{\text {eff }}\right)$. In compact notation, the thermodynamic average $\langle A\rangle_{T}$ of an operator $\hat{A}$ at temperature $T$ is obtained as a Monte Carlo average $\langle A\rangle_{T} \approx\left\langle A_{\mathrm{E}}\left(\left\{\mathbf{r}^{(s)}\right\}\right)\right\rangle_{W}$, where $A_{\mathrm{E}}$ is an estimator for $\hat{A}$ and $W$ is the sampling weight. The path-integral estimator for the derivative of the free energy in Eq. (3) is obtained most easily by substituting the path-integral representation (9) into the equation $-\beta d F / d \lambda=d \log Q(\lambda) / d \lambda$. This results in the thermodynamic estimator,

$$
-\beta \frac{d F_{\mathrm{TI}, \mathrm{TE}}(\lambda)}{d \lambda}=\sum_{i=1}^{N} \frac{d m_{i}}{d \lambda}\left(\frac{3 P}{2 m_{i}}-\beta \frac{d \Phi_{\mathrm{eff}}}{d m_{i}}\right),
$$

where

$$
\beta \frac{d \Phi_{\mathrm{eff}}}{d m_{i}}=\sum_{s=1}^{P}\left[\frac{P}{2 \hbar^{2} \beta}\left(\mathbf{r}_{i}^{(s)}-\mathbf{r}_{i}^{(s+1)}\right)^{2}+\frac{\beta}{P} \frac{d V_{\mathrm{TI}}\left(\mathbf{r}^{(s)}\right)}{d m_{i}}\right] .
$$

The problem with this estimator is the growth of its statistical error with $P$, preventing convergence to the quantum limit. A similar effect was observed for the thermodynamic estimator in the PA and was fixed by using the centroid virial estimator for $d F_{\mathrm{PA}}(\lambda) / d \lambda$ [14], which is a generalization of the centroid virial estimator for the kinetic energy [32, 33]. As in the PA used in Ref. [14], in the TI scheme this estimator can be derived by mass-scaling the coordinates [34] and subtracting the centroid coordinate $\mathbf{r}^{C}:=P^{-1} \sum_{s=1}^{P} \mathbf{r}^{(s)}$ in Eq. (9). This shifts the dependence on mass completely from the kinetic to the potential energy and the application of the equation $-\beta d F / d \lambda=d \log Q(\lambda) / d \lambda$ yields the desired TI centroid virial estimator:

$$
\begin{aligned}
&-\beta \frac{d F_{\mathrm{TI}, \mathrm{CVE}}(\lambda)}{d \lambda}=\frac{D}{2} \sum_{i=1}^{N} \frac{1}{m_{i}} \frac{d m_{i}(\lambda)}{d \lambda} \\
&-\frac{\beta}{P} \sum_{s=1}^{P}\left[\frac{d V_{\mathrm{eff}}\left(\left\{\mathbf{r}^{(s)}(\Delta \lambda)\right\}\right)}{d \Delta \lambda}-\frac{\hbar^{2}}{24}\left(\frac{\beta}{P}\right)^{2} \sum_{i=1}^{N} \frac{1}{m_{i}^{2}} \frac{d m_{i}}{d \lambda}\left(\frac{\partial V}{\partial \mathbf{r}_{i}}\left(\mathbf{r}_{i}^{(s)}\right)\right)^{2}\right], \\
& \mathbf{r}_{i}^{(s)}(\Delta \lambda):=\mathbf{r}_{i}^{C}+\left[\frac{m_{i}(\lambda)}{m_{i}(\lambda+\Delta \lambda)}\right]^{1 / 2}\left(\mathbf{r}_{i}^{(s)}-\mathbf{r}_{i}^{C}\right) .
\end{aligned}
$$


It is essential to evaluate the $\Delta \lambda$ derivative in the last expression by finite difference [34]. Evaluating this derivative analytically would result in expressions involving the gradient of $V_{\text {eff }}$ and requiring the second derivatives of $V$, which would eliminate the advantage of the TI PIMC. In general, the cost of evaluating the estimators can be made negligible in comparison with the cost of the random walk because, due to correlations between samples, it is not necessary to sample at each Monte Carlo step.

\subsection{Standard approach based on harmonic approximation}

In the numerical examples our results are compared with the standard approach [1] based on the "harmonic approximation." By harmonic approximation we mean an ensemble of the following three approximations: separability of rotations and vibrations, harmonic approximation for vibrations, and rigid rotor approximation for rotations. An overall EIE can be decomposed into elementary isotope effects (IEs), i.e., simple ratios of molecular partition functions: IE $:=Q_{l} / Q_{h}$. Within the harmonic approximation, the Teller-Redlich theorem yields [1]

$$
\mathrm{IE}_{\mathrm{HA}}=\mathrm{IE}_{\mathrm{HA}, T \rightarrow \infty} \prod_{n=1}^{3 N-6} \frac{x_{l, n}}{x_{h, n}} \frac{1-e^{-x_{h, n}}}{1-e^{x_{l, n}}} e^{-\left(x_{l, n}-x_{h, n}\right) / 2},
$$

where $n$ runs over the vibrational degrees of freedom, $x_{n}=\beta \hbar \omega_{n}$, and $\omega_{n}$ is the angular frequency of the $n$th vibration. The prefactor

$$
\mathrm{IE}_{\mathrm{HA}, T \rightarrow \infty}=\frac{s_{h}}{s_{l}} \prod_{i=1}^{N}\left(\frac{m_{l, i}}{m_{h, i}}\right)^{3 / 2}
$$

is the high temperature limit of $\mathrm{IE}_{\mathrm{HA}}$. The low temperature limit of $\mathrm{IE}_{\mathrm{HA}}$ (12) is

$$
\mathrm{IE}_{\mathrm{HA}, T \rightarrow 0}=\mathrm{IE}_{\mathrm{HA}, T \rightarrow \infty} \prod_{n=1}^{3 N-6} \frac{x_{l, n}}{x_{h, n}} e^{-\left(x_{l, n}-x_{h, n}\right) / 2} .
$$

The above expressions apply to nonlinear molecules, having $3 N-6$ vibrational degrees of freedom. Yet, these expressions remain valid in general when taking into account that the number of vibrations is zero for atoms and $3 N-5$ for linear molecules. 


\section{Numerical examples}

\subsection{Reactions}

Our methodology is tested numerically by evaluating the EIEs on two chemical reactions, the first EIE being the equilibrium constant for the isotopomerization reaction

$$
\mathrm{H}_{2}+\mathrm{D} \rightleftharpoons \mathrm{H}+\mathrm{HD},
$$

with the interesting part of the isotope effect being its deviation from the purely statistical value of 2 . Since the mass interpolation (44) allows changing masses of several atoms simultaneously, $\mathrm{EIE}_{1}$ can be calculated in a direct way as

$$
\mathrm{EIE}_{1}^{\text {direct }}=Q_{\mathrm{HD}+\mathrm{H}} / Q_{\mathrm{H}_{2}+\mathrm{D}} .
$$

For this calculation the free energy estimator must take into account a simultaneous transformation $\mathrm{H} \rightarrow \mathrm{D}$ in $\mathrm{H}_{2}$ and $\mathrm{D} \rightarrow-\rightarrow \mathrm{H}$ in D. Sometimes, however, it is useful to separate the contributions to the EIE from these two transformations and write:

$$
\mathrm{EIE}_{1}=\frac{Q_{\mathrm{HD}+\mathrm{H}} / Q_{\mathrm{H}_{2}+\mathrm{H}}}{Q_{\mathrm{H}_{2}+\mathrm{D}} / Q_{\mathrm{H}_{2}+\mathrm{H}}}
$$

where both the numerator and denominator were divided by $Q_{\mathrm{H}_{2}+\mathrm{H}}$. Finally, it is possible to write $\mathrm{EIE} \mathrm{(16)}$ ) as $\mathrm{EIE}=\left(Q_{\mathrm{HD}} / Q_{\mathrm{H}_{2}}\right)\left(Q_{\mathrm{H}} / Q_{\mathrm{D}}\right)$, where the first factor can be computed in a much simpler, 3-dimensional simulation (with one vibrational and two rotational coordinates) and the second factor evaluated analytically. We choose a more tedious, but more general approach expressed in Eqs. (16) or (17) and use nine Cartesian coordinates so that our methodology remains unchanged for unimolecular reactions (which have no asymptotic region with noninteracting reactants) and for large molecules (for which transformation to internal coordinates does not simplify the calculation). Last, a nine-dimensional calculation is a more stringent test of the methodology from Sec. 2.

The second EIE is an equilibrium constant for the isotopomerization reaction

$$
\mathrm{H}_{2}+\mathrm{D}_{2} \rightleftharpoons 2 \mathrm{HD}
$$

having a statistical value of 4 . Although this takes place on a different

potential energy surface, the EIE can be cast into a form suitable for the BKMP2 potential energy surface:

$$
\mathrm{EIE}_{2}=\frac{Q_{\mathrm{HD}+\mathrm{HD}}}{Q_{\mathrm{H}_{2}+\mathrm{D}_{2}}}=\frac{Q_{\mathrm{HD}} Q_{\mathrm{HD}}}{Q_{\mathrm{H}_{2}} Q_{\mathrm{D}_{2}}}=\frac{Q_{\mathrm{HD}+\mathrm{H}} / Q_{\mathrm{D}_{2}+\mathrm{H}}}{Q_{\mathrm{H}_{2}+\mathrm{H}} / Q_{\mathrm{HD}+\mathrm{H}}} .
$$


In the last step, both the numerator and denominator were multiplied by $Q_{\mathrm{H}}^{2}$. The second and third equalities hold because, in the low-pressure, low-concentration limit, the reactants and products are noninteracting in the asymptotic regions.

\subsection{Computational details}

All PIMC simulations were performed in Cartesian coordinates $\left(\mathbf{r}_{1}, \ldots, \mathbf{r}_{N}\right)$. The random walk employed the staging algorithm [35] to move half of the beads at once. The thermodynamic integral of Eq. (3) was evaluated with the Simpson method. The Trotter number was increased proportionally to the inverse temperature. Using the PA, in particular, the Trotter number was increased from $P=30$ at $1000 \mathrm{~K}$ to $P=160$ at $200 \mathrm{~K}$. Approximately the same convergence was obtained in the TI scheme when $P$ was increased from $P=8$ at $1000 \mathrm{~K}$ to $P=48$ at $200 \mathrm{~K}$. The simulations had an overall length of $10^{7}$ steps, of which $25 \%$ were a warm-up (i.e., equilibration of the system). Since the BKMP2 potential energy surface provides both energies and forces, the forces required in the TI scheme were computed analytically.

Harmonic approximation results were based on the frequencies from the BKMP2 PES, where the force constant was calculated as the numerical second derivative.

\subsection{Results}

Prior to calculating EIEs themselves, we analyzed convergence with respect to the Trotter number $P$ of the derivative of the free energy $F_{\mathrm{HD}}$ corresponding to the isotopic "alchemical" transformation $\mathbf{H}_{2}+\mathbf{H} \rightarrow \rightarrow$ $\mathbf{H D}+\mathbf{H}$ in the numerator of Eq. (17). Figure 1 demonstrates that both the PA and TI schemes converge to the same results for $P \rightarrow \infty$, yet the TI factorization converges to the quantum result for significantly smaller $P$ values, confirming the objective of our paper.

The advantages of the centroid virial estimators compared with thermodynamic estimators are shown in Fig. 2. Remarkably, the statistical errors behave similarly in the PA and TI scheme (except for the lowest $P$ values). In both factorizations, the statistical error of the thermodynamic estimator grows with $P$, whereas the error of the centroid virial estimator is approximately independent of $P$. This means that if the centroid virial estimator is used, the number of Monte Carlo samples need not be increased (at least not significantly) when $P$ is increased in order to reach the quantum limit. The

overall conclusion of Figs. [1-2 is that the optimal PIMC approach is using 


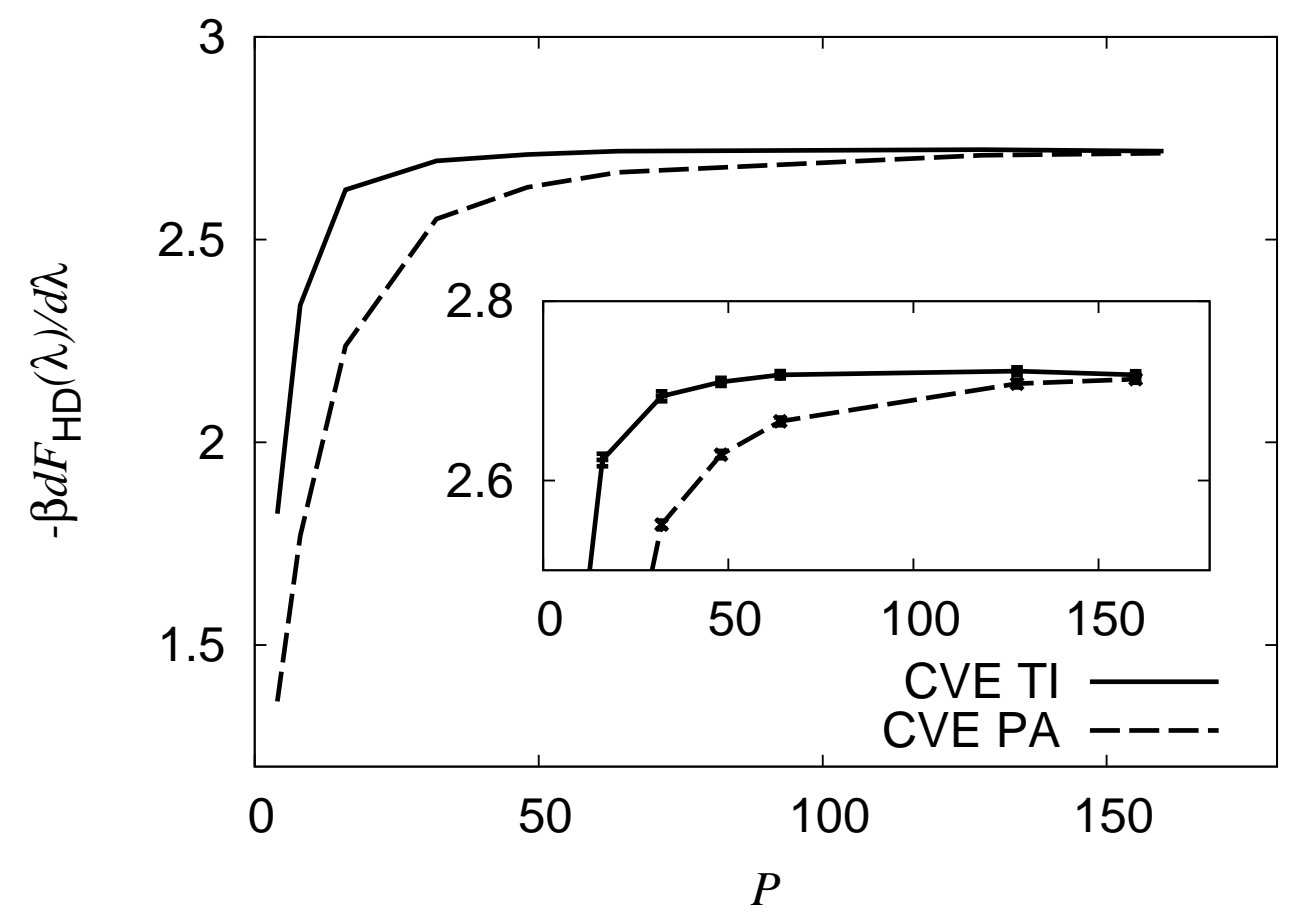

Figure 1: Free energy derivative as a function of the Trotter number $P$. The free energy derivative corresponds to the transformation $\mathrm{H}_{2}+\mathrm{H} \rightarrow \mathrm{HD}+\mathrm{H}$ at $T=200 \mathrm{~K}$ and is evaluated with the centroid virial estimator (CVE) at $\lambda=0.5$. The Takahashi-Imada (TI) factorization converges to the quantum limit much faster than the primitive approximation (PA). The inset shows a detail including statistical error bars. 
the TI factorization together with the centroid virial estimator: the former lowers the discretization error while the latter decreases the statistical error.

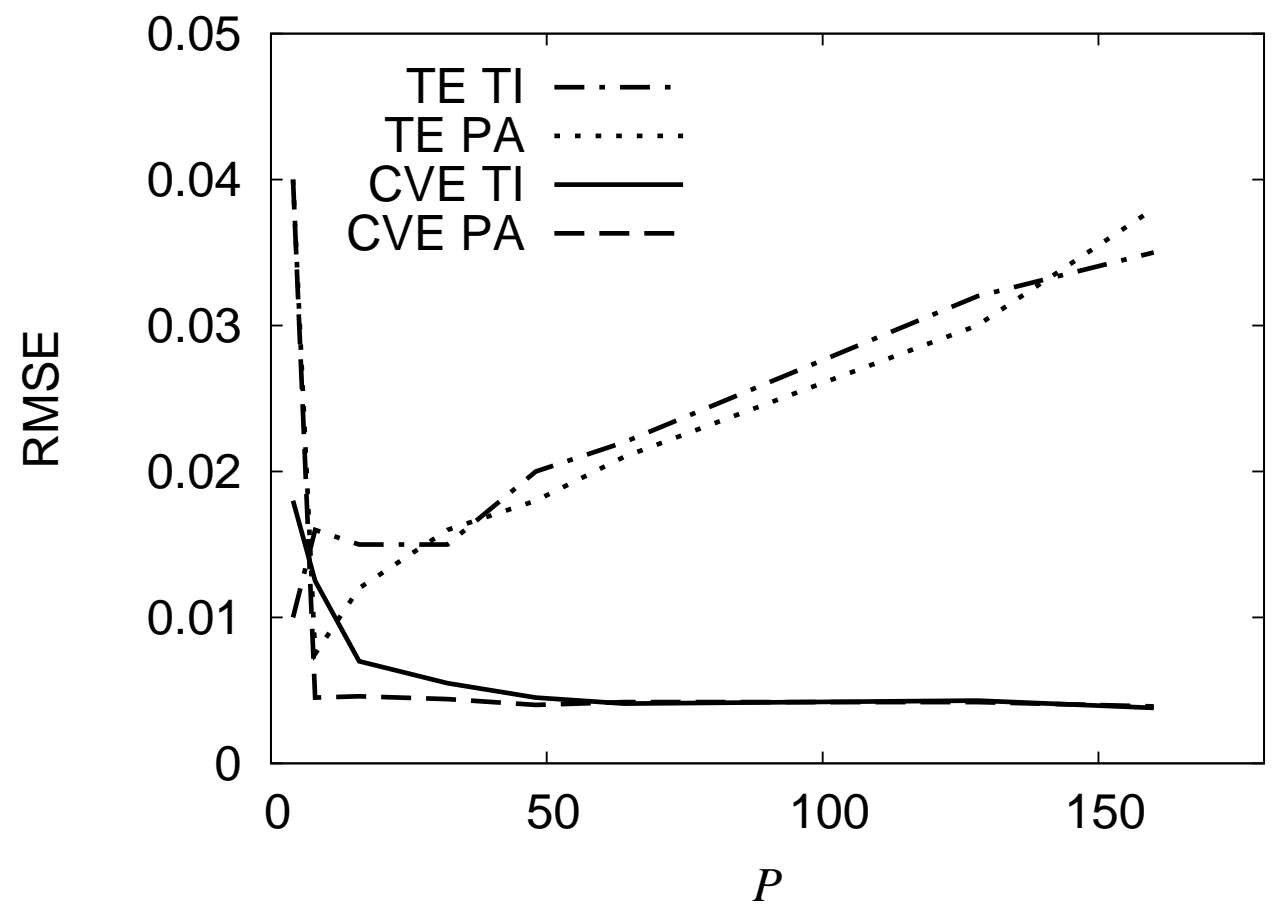

Figure 2: Statistical root mean square errors (RMSE) of various estimators for the free energy derivative $-\beta d F_{\mathrm{HD}}(\lambda) / d \lambda$ from Fig. 1 as a function of the Trotter number $P$. Both with the primitive approximation (PA) and Takahashi-Imada (TI) factorization, the RMSE is approximately independent of $P$ for the centroid-virial estimators (CVE), whereas it grows with $P$ for the thermodynamic estimators (TE).

Values of EIE $E_{1}$ at different temperatures with their statistical errors (computed by block-averaging [36]) are given in Table11and plotted in Fig. 3. Path integral results are compared with the harmonic approximation (12) and its high and low temperature limits [Eqs. (13) and (14)]. The table demonstrates that both the PA and TI factorizations, and both the thermodynamic and centroid virial estimators converge to the same result, which differs from the harmonic approximation. The only, yet crucial differences, among the four PIMC results are in the required value of $P$ (which is lower in the TI scheme than in the PA) and in the statistical error (which is much lower for the centroid virial than for the thermodynamic estimators). Surprisingly, the 
PI correction to the harmonic approximation of the EIE is rather small; this was observed before, e.g., in the EIE on three sigmatropic hydrogen shift reactions [3]. The reason is that the quantum harmonic approximation works rather well in rigid molecules. The PI corrections would be more pronounced in the EIE in floppy and other very anharmonic systems, or in the kinetic isotope effect on reactions with significant tunneling contribution.

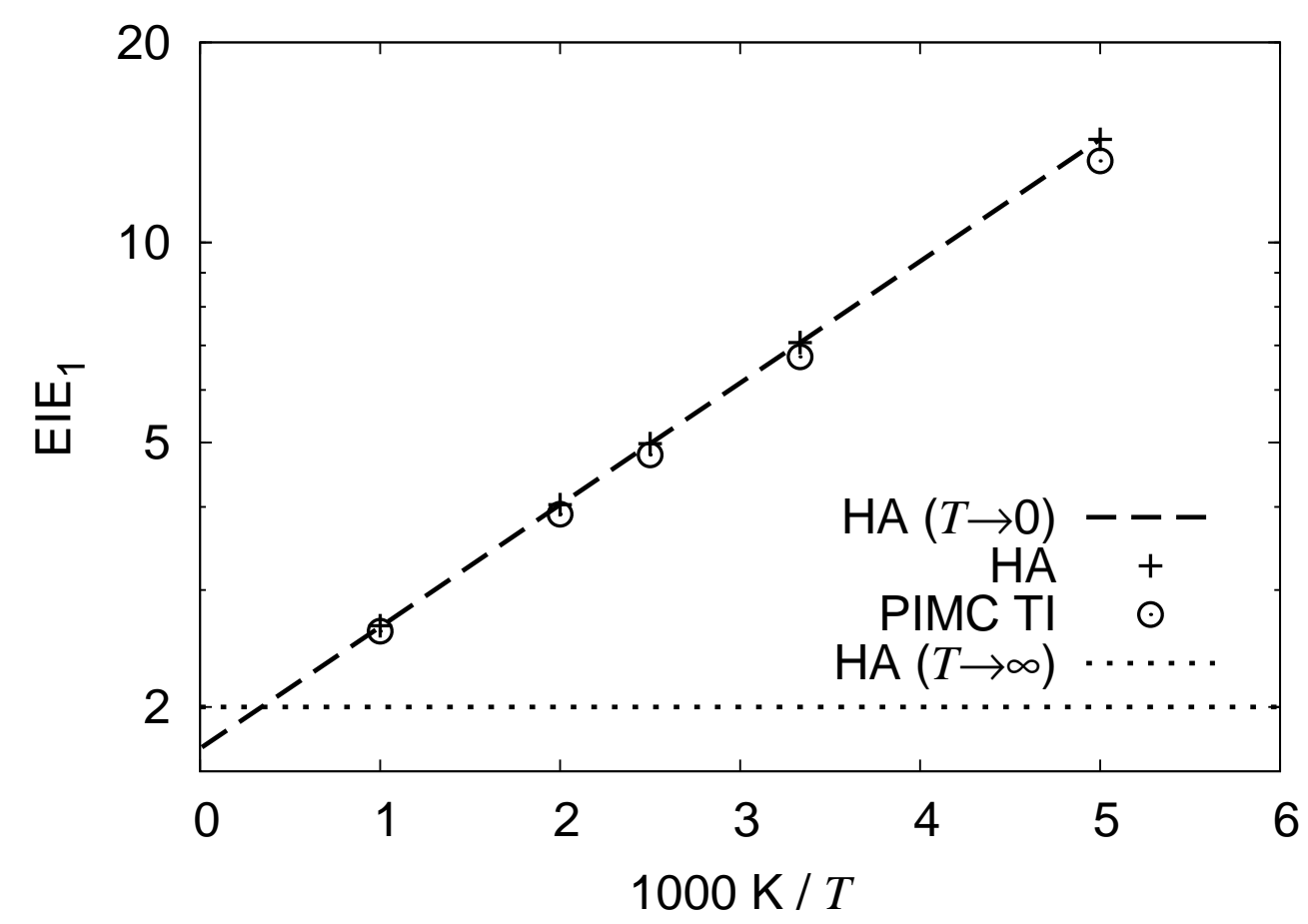

Figure 3: Temperature dependence of the equilibrium isotope effect $\mathrm{EIE}_{1}$ (17). Comparison between the harmonic approximation (HA) and the present PIMC method using the Takahashi-Imada factorization (PIMC TI).

These differences are reflected in the simulation lengths. Table 2 shows speedups achieved using different methods in comparison with the simulation using the PA and the thermodynamic estimator. The speedup $f$ is defined as

$$
f_{\text {method }}:=\left(\frac{\sigma_{\mathrm{PA}+\mathrm{TE}}}{\sigma_{\text {method }}}\right)^{2} \frac{t_{\mathrm{PA}+\mathrm{TE}}}{t_{\mathrm{method}}},
$$

where "method" stands for the factorization and estimator used $(\mathrm{TE}=$ ther- 
Table 1: Equilibrium isotope effect EIE $_{1}$ (17) at several temperatures. Table compares results of PIMC calculations using either the primitive approximation (PA) or TakahashiImada (TI) factorizations, and either the thermodynamic (TE) or centroid-virial (CVE) estimators. $P$ is the Trotter number and the statistical error is shown in parentheses. Results of the direct PIMC approach (16) and of the harmonic approximation (HA) are shown as well.

\begin{tabular}{ccccccccc}
\hline \hline$T(\mathrm{~K})$ & \multicolumn{3}{c}{ PIMC PA } & \multicolumn{4}{c}{ PIMC TI } & \multirow{2}{*}{ HA } \\
\cline { 2 - 7 } & TE & CVE & $P$ & TE & CVE & CVE direct & $P$ & \\
\hline 200 & $13.1(3)$ & $13.24(3)$ & 160 & $13.3(2)$ & $13.27(3)$ & $13.19(4)$ & 48 & 14.29 \\
300 & $6.82(8)$ & $6.72(1)$ & 110 & $6.66(5)$ & $6.73(1)$ & $6.70(1)$ & 32 & 7.07 \\
400 & $4.84(4)$ & $4.778(2)$ & 80 & $4.78(2)$ & $4.788(3)$ & $4.770(3)$ & 24 & 4.98 \\
500 & $3.91(2)$ & $3.896(1)$ & 60 & $3.91(1)$ & $3.905(2)$ & $3.892(2)$ & 18 & 4.03 \\
1000 & $2.605(9)$ & $2.6018(3)$ & 30 & $2.600(7)$ & $2.6036(4)$ & $2.5966(4)$ & 8 & 2.65 \\
\hline \hline
\end{tabular}

modynamic estimator), $t$ is the CPU time taken by the simulation, and $\sigma$ is the statistical error achieved. The number of beads, $P$, was chosen so that the discretization error was roughly the same for the PA and TI factorization. The thermodynamic and centroid virial estimators were computed together in a single simulation, which is the reason why the factor involving statistical errors appears in Eq. (20). Table 2 shows that TI factorization alone accelerates simulations by a factor between 5 and 10. If the TI factorization is augmented with the centroid virial estimator, the overall acceleration is 2002000 fold. Surprisingly, the speedups due to the use of the centroid virial estimator at low temperatures are smaller than at high temperatures; this could be because at high temperatures the system accesses flatter regions of the PES and because the centroid virial estimator yields (in the PA) the exact result for the free particle without any sampling.

Results for $\mathrm{EIE}_{2}$ are displayed in Table 3, Everything said about EIE $\mathrm{E}_{1}$ holds here, and in addition the PIMC results also agree with the harmonic approximation. The reason is probably the cancellation of errors due to the harmonic approximation between the ratios $Q_{\mathrm{D}_{2}} / Q_{\mathrm{HD}}$ and $Q_{\mathrm{HD}} / Q_{\mathrm{H}_{2}}$.

The results of $\mathrm{EIE}_{2}$ were also found in a reasonable agreement with the experimental data of Urey et al. [37]. Those values are shown in Table 3 (in the column EXP.) and were obtained by linear regression of the dependence of $\mathrm{EIE}_{2}$ from Ref. [37] as a function of $1 / T$ between the temperatures of $25^{\circ} \mathrm{C}$ and $468^{\circ} \mathrm{C}$; the datapoint 
Table 2: Speedup achieved in computing EIE 1 (17) by various methods in comparison with the time taken by the primitive approximation (PA) with the thermodynamic estimator (TE). E.g., at $1000 \mathrm{~K}$, the calculation with the Takahashi-Imada (TI) factorization and the centroid-virial estimator (CVE) is 2125 times faster.

\begin{tabular}{ccccc}
\hline \hline$T(\mathrm{~K})$ & \multicolumn{2}{c}{ PA } & \multicolumn{2}{c}{ TI } \\
& TE & CVE & TE & CVE \\
\hline 200 & 1 & 148 & 9 & 285 \\
300 & 1 & 159 & 7 & 476 \\
400 & 1 & 347 & 9 & 582 \\
500 & 1 & 370 & 10 & 403 \\
1000 & 1 & 865 & 5 & 2125 \\
\hline \hline
\end{tabular}

at the intermediate temperature from Ref. [37] was omitted as too uncertain and the average of the two available results at $468^{\circ} \mathrm{C}$ was used.

The speedups achieved with the TI factorization and centroid virial estimator are shown in Table 4 and have the same order of magnitude as those for $\mathrm{EIE}_{1}$ in Table 2 ,

Table 3: Equilibrium isotope effect $\mathrm{EIE}_{2}$ (19) at several temperatures. See caption of Table 1 for details. Column EXP. contains values obtained by linear regression of experimental results [37].

\begin{tabular}{ccccccccc}
\hline \hline \multirow{2}{*}{$T(\mathrm{~K})$} & \multicolumn{3}{c}{ PIMC PA } & \multicolumn{3}{c}{ PIMC TI } & \multirow{2}{*}{ HA } & EXP. \\
\cline { 2 - 7 } & TE & CVE & $P$ & TE & CVE & $P$ & & \\
\hline 200 & $2.83(6)$ & $2.85(1)$ & 160 & $2.85(3)$ & $2.84(1)$ & 48 & 2.86 & 2.89 \\
300 & $3.31(3)$ & $3.256(4)$ & 110 & $3.24(2)$ & $3.251(4)$ & 32 & 3.26 & 3.28 \\
400 & $3.54(3)$ & $3.473(2)$ & 80 & $3.49(1)$ & $3.474(3)$ & 24 & 3.49 & 3.48 \\
500 & $3.63(2)$ & $3.618(2)$ & 60 & $3.60(1)$ & $3.616(2)$ & 18 & 3.63 & 3.60 \\
1000 & $3.89(1)$ & $3.897(1)$ & 30 & $3.90(1)$ & $3.894(1)$ & 8 & 3.90 & 3.83 \\
\hline \hline
\end{tabular}

As mentioned above, both $\mathrm{EIE}_{1}$ and $\mathrm{EIE}_{2}$ are equilibrium constants of isotopomerization reactions, hence the present method can be viewed as complementary to the PIMC method for calculating the temperature dependence of the equilibrium constant described in Ref. [38]. In Ref. [38], the equilibrium constant $K(T)$ at temperature $T$ is obtained by thermodynamic integration with respect to the inverse temperature from the equilibrium constant $K\left(T_{0}\right)$ at temperature $T_{0}$. In isotopomerization reactions, $K\left(T_{0} \rightarrow \infty\right)$ at high tem- 
Table 4: Speedup achieved in computing $\mathrm{EIE}_{2}$ (19) by various methods. See caption of Table 2 for details.

\begin{tabular}{ccccc}
\hline \hline$T(\mathrm{~K})$ & \multicolumn{2}{c}{ PA } & \multicolumn{2}{c}{ TI } \\
& TE & CVE & TE & CVE \\
\hline 200 & 1 & 54 & 10 & 146 \\
300 & 1 & 63 & 10 & 229 \\
400 & 1 & 150 & 9 & 233 \\
500 & 1 & 154 & 9 & 217 \\
1000 & 1 & 243 & 5 & 1012 \\
\hline \hline
\end{tabular}

peratures is simply given by the ratio of the symmetry numbers, which follows easily from Eq. (13).

Note also that the second alternative form of the $\mathrm{EIE}_{2}$ in Eq. (19) involves separate reactant or product molecules, and can be used with molecular potential energy surfaces if the full reactive potential energy surface is not available.

\section{Conclusions}

Using two isotopomerization reactions, we have demonstrated that TI factorization significantly decreases the Trotter number required for convergence of PIMC calculations of EIEs to the quantum limit. This leads to significant acceleration of the calculations whether forces are available in an analytical form, or must be computed by finite differences from energies. Similar acceleration was observed in Refs. [5, 29]. Moreover, we have observed that the large difference in the statistical convergence of the thermodynamic and centroid virial estimators, well known from the PIMC calculations based on the PA, reappears in the TI scheme. Unlike the thermodynamic estimator, the centroid virial estimator has a statistical error independent of the Trotter number. As shown in Sec. 3, this can easily accelerate calculations by orders of magnitude.

The TI scheme applies, unfortunately, only to the diagonal matrix elements of the density matrix. If the off-diagonal elements are needed, as in computing the momentum distribution functions, alternative high order factorizations are required [29]. An example is the Suzuki-Chin factorization [39, 40]. Indeed, one of us is presently exploring how this factorization could be used to efficiently compute the kinetic iso- 
tope effects as opposed to the EIE. In calculations of diagonal elements, several Chin factorizations [20] may converge faster than the TI splitting although they have the same order in general [5]. Yet, the TI scheme has one decisive advantage, which is its simplicity. As demonstrated here, the TI scheme is easily implemented in existing codes based on the PA: the implementation simply replaces the original potential $V$ by the TI effective potential $V_{\text {eff. }}$ Unlike some more sophisticated factorizations, the TI scheme treats all imaginary time slices equally. In conclusion, we believe that the TI factorization will find more interesting applications in path integral simulations of equilibrium and nonequilibrium quantum effects.

\section{Acknowledgments}

This research was supported by the Swiss NSF Grant No. 200021_124936/1 to J.V. and by the EPFL. We thank Matthew Wodrich for a careful reading of the manuscript.

\section{References}

[1] M. Wolfsberg, W. Alexander van Hook, P. Paneth, L.P.N. Rebelo, Isotope Effects in the Chemical, Geological, and Bio Sciences, Springer, Dordrecht Heidelberg London New York, 2010.

[2] V.A. Lynch, S.L. Mielke, D.G. Truhlar, J. Phys. Chem. A 109 (2005) 10092.

[3] T. Zimmermann, J. Vaníček, J. Chem. Phys. 131 (2009) 024111.

[4] T. Zimmermann, J. Vaníček, J. Mol. Modeling 16 (2010) 01779.

[5] A. Azuri, H. Engel, D. Doron, D.T. Major, J. Chem. Theory Comput. 7 (2011) 1273.

[6] A. Perez, O.A. von Lilienfeld, J. Chem. Theory Comput. 7 (2011) 2358.

[7] M. Ceriotti, D.E. Manolopoulos, Phys. Rev. Lett. 109 (2012) 100604.

[8] M. Ceriotti, T.E. Markland, J. Chem. Phys. 138 (2013) 014112.

[9] R. Feynman, A. Hibbs, Quantum Mechanics and Path Integrals, McGraw-Hill, 1965. 
[10] H. Kleinert, Path Integrals in Quantum Mechanics, Statistics, Polymer Physics and Financial Markets, World Scientific Publishing Co. Pte. Ltd., 2004.

[11] D.M. Ceperley, Rev. Mod. Phys. 67 (1995) 279.

[12] T. Yamamoto, W.H. Miller, J. Chem. Phys. 122 (2005) 044106.

[13] J. Vaníček, W.H. Miller, J.F. Castillo, F.J. Aoiz, J. Chem. Phys. 123 (2005) 054108.

[14] J. Vaníček, W.H. Miller, J. Chem. Phys. 127 (2007) 114309.

[15] W.H. Miller, Y. Zhao, M. Ceotto, S. Yang, J. Chem. Phys. 119 (2003) 1329.

[16] S.L. Mielke, D.G. Truhlar, Chem. Phys. Lett. 378 (2003) 317.

[17] R.D. Coalson, J. Chem. Phys. 85 (1986) 926.

[18] J.K. Hwang, A. Warshel, J. Phys. C 97 (1993) 10053.

[19] M. Takahashi, M. Imada, J. Phys. Soc. Jpn. 53 (1984) 3765.

[20] A. Chin, Phys. Rev. E 69 (2004) 046118.

[21] A.I. Boothroyd, W.J. Keogh, P.G. Martin, M. R. Peterson, J. Chem. Phys. 104 (1996) 7139.

[22] J.E. Mayer, M.G. Mayer, Statistical Mechanics, Wiley, New York, 1977.

[23] D. Frenkel, B. Smit, Understanding Molecular Simulation, Academic Press, 2002.

[24] M. Wolfsberg, Acc. Chem. Res. 5 (1972) 225.

[25] H. F. Trotter, Proc. Amer. Math. Soc. 10 (1959) 545.

[26] S. Jang, S. Jang, G.A. Voth, J. Chem. Phys. 115 (2001) 7832.

[27] L. Brualla, K. Sakkos, J. Boronat, J. Casulleras, J. Chem. Phys. 121, 636 (2004).

[28] T. Yamamoto, J. Chem. Phys. 123 (2005) 104101. 
[29] A. Perez, M.E. Tuckerman, J. Chem. Phys. 135 (2011) 064104.

[30] R. Hellmann, E. Bich, E. Vogel, V. Vesovic, Phys. Chem. Chem. Phys. 13 (2011) 13749.

[31] M. Ceriotti, G.A.R. Brain, O. Riordan, D.E. Manolopoulos, Proc. R. Soc. A. 468 (2012) 2.

[32] M.F. Herman, E.J. Bruskin, B.J. Berne, J. Chem. Phys. 76 (1982) 5150.

[33] M. Parrinello, A. Rahman, J. Chem. Phys. 80 (1984) 860.

[34] C. Predescu, Phys. Rev. E, 70 (2004) 066705.

[35] M. Sprik, M.L. Klein, D. Chandler, Phys. Rev. B 31 (1985) 4234.

[36] H. Flyvbjerg, H.G. Petersen, J. Chem. Phys. 91 (1989) 461.

[37] D. Rittenberg, W. Bleakney, H.C. Urey, J. Chem. Phys. 2 (1934) 48.

[38] M. Buchowiecki, Chem. Phys. Lett. 531 (2012) 202.

[39] M. Suzuki, Phys. Lett. A 201 (1995) 425.

[40] S.A. Chin, Phys. Lett. A 226 (1997) 344. 